\title{
In reply: Is sugammadex alone enough to cause anaphylaxis?
}

\author{
Masakazu Yamaoka ${ }^{1}$ \\ Received: 6 January 2018 / Accepted: 8 January 2018 / Published online: 19 January 2018 \\ (c) Japanese Society of Anesthesiologists 2018
}

Keywords Sugammadex $\cdot$ Rocuronium-sugammadex complex $\cdot$ Anaphylaxis $\cdot$ Cesarean section

To the Editor:

We would like to thank for fruitful comments on my article "A suspected case of rocuronium-sugammadex complexinduced anaphylactic shock after cesarean section" [1].

We are wondering if they believe that sugammadex alone caused the anaphylaxis in our case? We, at first, considered sugammadex was the causative agent because our patient fell into anaphylactic shock following sugammadex administration and extubation. However, a later intradermal skin test with undiluted rocuronium 40 min after sugammadex tests revealed a strong positive reaction. This test made her vital signs fall into a shock state. While the technique of our skin test might be inappropriate, we do not think our rocuronium test was false positive because the reaction was too severe. Then, our subsequent skin test with premixed sugammadex-rocuronium complex was positive. Comprehensively, we think the best explanation for this case is that rocuronium-sugammadex complex, not rocuronium alone or sugammadex alone, induced anaphylaxis.
We are very thankful to them for introducing the wellestablished report of the first case of sugammadex-induced anaphylaxis validated by the basophil activation test (BAT) [2]. Additional in vitro tests like BAT must enable us to confirm the causative agent without exposing the patient to further risk.

\section{Compliance with ethical standards}

Conflict of interest Masakazu Yamaoka has no conflict of interest.

\section{References}

1. Yamaoka M, Deguchi M, Ninomiya K, Kurasako T, Matsumoto M. A suspected case of rocuronium-sugammadex complexinduced anaphylactic shock after cesarean section. J Anesth. 2017;31:148-51.

2. Takazawa T, Horiuchi T, Yoshida N, Yokohama A, Saito S. Flow cytometric investigation of sugammadex-induced anaphylaxis. $\mathrm{Br}$ J Anaesth. 2015;114:858-9.

This reply refers to the article available at https://doi.org/10.1007/ s00540-018-2453-4.

Masakazu Yamaoka

mskz16@yahoo.co.jp

1 Department of Anesthesiology, Japanese Red Cross Society Himeji Hospital, 1-12-1 Shimoteno, Himeji, Japan 